$\%$ A L. Ingber

\% T Path-integral Riemannian contributions to nuclear Schrödinger equation

$\%$ J Phys. Rev. D

$\%$ V 29

$\% \mathrm{P} 1171-1174$

$\% \mathrm{D} 1984$

\title{
Path-integral Riemannian contributions to nuclear Schr̈odinger equation
}

\author{
Lester Ingber \\ Physical Studies Institute, Drawer W, Solana Beach, California 92075 \\ and Institute for Pure and Applied Physical Sciences, \\ University of California San Diego, La Jolla, California 92093
}

(Received 28 July 1983)

Several studies in quantum mechanics and statistical mechanics have formally established that nonflat metrics induce a difference in the potential used to define the path-integral Lagrangian from that used to define the differential Schrodinger Hamiltonian. A recent study has described a statistical mechanical biophysical system in which this effect is large enough to be measurable. This study demonstrates that the nucleon-nucleon velocity-dependent interaction derived from meson exchanges is a quantum mechanical system in which this effect is also large enough to be measurable.

PACS numbers 1983: 13.75.Cs, 03.65.Db, 21.30.+y, 02.50.+s

\section{INTRODUCTION}

In the last few years, several investigators have noted that the potential contribution in the differential Hamiltonian operator of the Schrodinger equation differs from the corresponding potential contribution to the path-integral Lagrangian, when the metric is non-flat [1-10]. Similar differences occur in statistical mechanics, between the differential Fokker-Planck equation and the Onsager-Machlup Lagrangian, extended to systems with nonlinear drift and nonconstant diffusion [11-14].

All these authors have noted that these differences are as yet untested, in that these Riemannian "corrections" are too small to be measurable in most physical systems.

However, recently a statistical mechanical system, the statistical mechanics of neocortical interactions $[15,16]$, has been shown to possess such corrections that are large enough to be measurable within ranges of current empirical values of synaptic and neuronal parameters. Although the scalar Riemannian curvature and covariant divergence of the drift terms are within a few percent of the bulk of the Hamiltonian and Lagrangian, the neocortical system is not quite yet sufficiently experimentally defined to enable these calculations to predict actual changes in stability and information processing. The statistical mechanics of financial markets also has been shown to have similar mathematical behavior [17], and here these corrections further highlight the importance of dealing with the Stratonovich versus the Ito prescriptions, i.e., the midpoint versus the prepoint discretization, respectively [8]. This system awaits definitive fits to market data to establish the actual importance of these differences.

Also recently, a quantum mechanical system, nonrelativistic nuclear forces, has been shown to possess Riemannian corrections that are indeed large enough to be measurable [18]. These corrections are enhanced because of the standard methodology, by which parameters of meson-exchange nuclear forces are fitted by the Schrodinger equation to empirical scattering and deuteron data; but nuclear matter must be described by a time-folded path-integral-type formalism to account for ladder and self-energy effects among particles and holes relative to the Fermi surface [18,19]. A key point in this argument is that any path-integral-type derivation of the $K$-matrix--i.e., involving the time-folding of a differential propagator--that begins with a two-body momentum-dependent two-nucleon interaction, gives rise to a calculable difference in the effective potential compared with the potential used in the two-nucleon Schrodinger equation. Since the $K$-matrix itself is not exactly reducible to a Schrodinger partial differential equation, this argument is invoked at the earlier stage first defining the many-body operator containing $V_{K}$, which of 
course is widely assumed to at least model $V_{\psi}$, before the self-energy and ladder partial sums are taken. In any such derivation, the modification of each kinetic energy term by its momentum-dependent potential defines a non-flat metric giving rise to this effect.

In a previous paper [18], the curvature correction was argued to be measurable by virtue of simply considering just two terms out of 159, in "momentum-transfer-space," that have been used to fit nuclear force data $[19,20]$. This paper includes the full potential, and therefore goes another step forward to quantitatively demonstrate that the curvature scalar is consistently at least a few percent as large as the nucleon-nucleon potential itself, over the nonrelativistic region of interaction. Even though it is interesting and relevant to path-integral formalism that this effect is large in some physical systems, an important point is that, although this curvature scalar contribution might be considered to be absorbed by the nucleon-nucleon potential parameters (e.g., coupling constants and masses of exchanged mesons) by their fit to scattering and deuteron data, this is not true in practice [18]. These parameters have always been fit using the Schrodinger equation, e.g., which contains the curvature scalar in its potential, but the path-integral-type derivation of the nuclear matter binding energy does not contain this term in the potential appearing in the Lagrangian, and therefore a contribution $\sim 1 \mathrm{Mev}$ to the theoretical binding energy is obtained which is larger than the empirical error in the binding energy, i.e., the volume term in the "Bethe-Weis̈acker semi-empirical mass formula."

Section II summarizes the basic formalism. Section III summarizes the numerical calculations.

\section{BASIC FORMALISM}

The path integral for nonrelativistic interactions can be written for the scalar amplitude $\Psi, \Psi$ invariant under coordinate transformations.

$$
\begin{aligned}
\Psi & =\lim _{N \rightarrow \infty}\left(\frac{\mu_{m}}{2 \pi i \varepsilon}\right)^{n(N+1) / 2} \int \cdots \int \prod_{s=1}^{N} g^{1 / 2} \Delta^{z} \exp \left(i \int_{(s-1) \varepsilon}^{s \varepsilon} L\right), \\
L & =\frac{1}{2 \mu_{m}} g_{i j} \dot{x}^{i} \dot{x}^{j}-\hat{v}_{\Psi}(x), \\
& =\frac{1}{2 \mu_{m}} \eta_{i j} \dot{x}^{i} \dot{x}^{j}-\hat{V}_{\Psi}(\dot{x}, x),
\end{aligned}
$$

where $\eta_{i j}$ is the flat-space metric in the presence of velocity-dependent potential $\hat{V}, g=\operatorname{det}\left(g_{i j}\right), g_{i j}$ is the proper metric of the space, $\mu_{m}=m / 2$ is the reduced average nucleon mass, and $\Delta^{z}$ is a normalization to be further specified subsequently. In the nucleon-nucleon problem, $-\hat{V}$ is the interaction Lagrangian, e.g., defined by the Born terms of meson-exchange nucleon-nucleon amplitudes.

This is clearly very phenomenological, despite the fact that for many decades there has been moderate to good numerical success in so defining $\hat{V}$ : There are many prescriptions for $\hat{V}$, e.g., dispersion relations for multiple meson-exchanges [21], and quark models or tower graphs from regge analysis for the core region inside $2 \mathrm{Gev}^{-1}=0.395 \mathrm{fm}$ [22-24]. Therefore, theory has a long way to go to explain a unique $\hat{V}$, if one even exists. However, the effect considered here arises from any momentum-dependent contributions to $\hat{V}$, and it is clear that at the the least, such momentum dependence arises from nucleon spinors and one-meson-exchange diagrams [19].

For very practical reasons, to facilitate fitting of experimental data and to formulate the nuclear matter problem, a Schrodinger equation must be written corresponding to $\Psi$. To specify the momentum operators, first a covariant Schrodinger equation is required for the scalar wave-function $\Psi=g^{-1 / 4} \psi$, where $\psi$ is the conventional nonrelativistic wave-function.

$$
\begin{aligned}
i \partial_{t} \Psi=H_{\Psi} \Psi & =-\frac{1}{2 \mu_{m}}\left(g^{i j} \Psi_{; j}\right)_{; i}+V_{\Psi} \Psi \\
& \equiv\left[-\frac{1}{2 \mu_{m}} g^{-1 / 2} \partial_{i} g^{1 / 2} g^{i j} \partial_{j}+V_{\Psi}\right] \Psi,
\end{aligned}
$$




$$
\partial_{i}[\cdots]=\partial[\cdots] / \partial x^{i}
$$

where $g^{i j}=\left(g_{i j}\right)^{-1}, H_{\Psi}$ is the differential Hamiltonian operator, and $V_{\Psi}=V_{\Psi}(x)$. As established by previous studies [1-6,8,11-14,25], the path-integral Lagrangian corresponding to $H_{\Psi}$ is

$$
L_{\Psi}=\frac{1}{2 \mu_{m}} g_{i j} \dot{x}^{i} \dot{x}^{j}-V_{\Psi}-R /\left(6 \mu_{m}\right) .
$$

Note that the covariant divergence of the gradient $\left(g^{i j} \Psi_{; j}\right)_{; i}$ reduces to the ordinary Laplacian $\nabla_{x}^{2} \Psi$ in orthogonal coordinates, and that $R$ is defined here to have the same sign as the Gaussian curvature.

$g_{i j}$ is defined here by the effect of $V_{\psi}\left(r,-\nabla_{r}^{2}\right)$ on the flat-space metric $\eta_{i j}$ in $H_{\psi}$. Considering a momentum-dependent central potential, e.g., $S$-states,

$$
\begin{aligned}
& i \partial_{t} \psi=H_{\psi} \psi=\left[-\frac{1}{m} g^{-1 / 4} \partial_{i} g^{1 / 2} g^{i j} \partial_{j} g^{-1 / 4}+v_{s}-R /\left(12 \mu_{m}\right)\right] \psi \\
& \equiv\left[-\frac{1}{m} \eta^{-1 / 4} \partial_{i} \eta^{1 / 2} \eta^{i j} \partial_{j} \eta^{-1 / 4}+V_{\psi}{ }^{\prime}\right] \psi \equiv\left[\frac{1}{m} p^{2}+V_{\psi}\right] \psi, \\
& g^{i j}=(1+W) \eta^{i j}, \eta=\operatorname{det}\left(\eta_{i j}\right), \\
& V_{\psi}\left(r, p^{2}\right)=v_{s}(r)-R(r) /\left(12 \mu_{m}\right)+\left\{p^{2} W(r)\right\} / m, \\
& \left\{p^{2} W\right\}=W p^{2}+Y^{i}[W, \eta] \partial_{i}+Z[W, \eta] .
\end{aligned}
$$

The functions $W, Y$ and $Z$ are directly, albeit tediously calculated in Eq. (4), from Eq. (2) with $\Psi=g^{-1 / 4} \psi: g^{i j}$ is defined by the velocity-dependent potential $W ; V_{\psi}\left(r, p^{2}\right)$ is defined by expanding and absorbing all terms from the second-order differential operator in $g^{i j}$-space which do not contribute to $\nabla^{2} \psi$ in flat $\eta^{i j}$-space, via the intermediate step involving $V_{\psi}{ }^{\prime}$ (e.g., factors of $\eta$ are passed through to the LHS of differential operators); this also defines the calculation of terms $\left\{p^{2} W\right\}$ which contribute to the sum of the usual static potential $v_{s}$ plus the scalar curvature contribution. The terms in $W, Y$ and $Z$ will be included in future nucleon-nucleon fits and nuclear matter calculations; similar terms were included in previous calculations $[19,20]$. However, the scalar curvature term contribution is relatively independent of the fitting process, and it is this contribution that is being analyzed in this paper.

This choice of $V_{\psi}$ leaves the path-integral Lagrangian for $\psi, L_{\psi}$ (to be associated with $V_{K}$ and the $K$-matrix), $R$-free and "gauge"-free: $R$ does not appear in the classical $L_{\psi}$, resulting in $-R /\left(12 \mu_{m}\right)=-R /(6 m)$ in $H_{\psi}$ instead of $-R /\left(6 \mu_{m}\right)$ in $L_{\Psi}$. The covariance of $\left(g^{i j} \Psi_{; j}\right)_{; i}$ dictates the ordering of $p^{2}$ and $W$ in $H_{\psi}$, and also keeps $L_{\psi}$ a quadratic form in $\dot{x}^{i} \dot{x}_{i}$, i.e., not in $\left(\dot{x}^{i}-w^{i}\right)\left(\dot{x}_{i}-w_{i}\right)$ for some nonzero $w^{i}$. Note that $|W|<1$ for all reasonable two-nucleon potentials in the nonrelativistic region. In this form, the scattering problem, the deuteron and nuclear matter can be solved straightforwardly, as demonstrated previously [19,20].

Much mystery is taken out of this calculation by recognizing that the differential $H_{\Psi}$ is calculated from $L_{\Psi}$ by considering fluctuations $y^{i}$ about the geodesics in normal coordinates.

$$
\begin{aligned}
& g_{i j}(y)=g_{i j}(0)-\frac{1}{6}\left[R_{i k j l}(0)+R_{j k i l}(0)\right] y^{k} y^{l}+\cdots, \\
& g(y)=g(0)\left(1-\frac{1}{3} R_{i j} y^{i} y^{j}+\cdots\right), \\
& R_{k j i l}=\frac{1}{2}\left(g_{i j, k l}-g_{j k, i l}-g_{i l, j k}+g_{j l, i k}\right)+g_{m n}\left(\Gamma_{i k}^{m} \Gamma_{j l}^{n}-\Gamma_{i l}^{m} \Gamma_{j k}^{n}\right), \\
& \Gamma_{j k}^{i}=g^{l i}[j k, l]=\frac{1}{2} g^{l i}\left(g_{j l, k}+g_{k l, j}-g_{j k, l}\right), \\
& R=g^{j l} R_{j l}=g^{j l} g^{i k} R_{i j k l},
\end{aligned}
$$




$$
[\cdots]_{, i}=\partial[\cdots] / \partial x^{i},
$$

Therefore, if $L_{\Psi}$ is $R$-free, then $H_{\Psi}$ contains a term of $-R /\left(6 \mu_{m}\right)$. If $L_{\psi}$ is $R$-free, then $H_{\psi}$ contains a term $-R /\left(12 \mu_{m}\right)$ due to the $g^{1 / 4}$ normalization factor. This source of $R$ in $\Psi$ also summarizes why it is possible to have an "extra" normalization of $\Delta^{z}$ in the path integral, as long as $L_{\Psi}$ is redefined as $L_{\Psi^{\prime}}=L_{\Psi}+z R /\left(6 \mu_{m}\right)$ [4]. With this consistent choice of $\Psi^{\prime}$ and $\Delta^{z}$, the path-integral solution for $\Psi$ is independent of the numerical value of $z$. I.e., in Eq. (1), $L$ should be replaced by $L \psi^{\prime}$. Here, $z$ is taken to be zero. In statistical mechanics, in two dimensions where one component of the curvature tensor suffices to specify the curvature scalar, it has also been noted that if $H_{\Psi}$ is kept $R$-free by redefining the potential $V, \Psi$ now representing the scalar probability function, then the $R$ term in $L_{\Psi}$ can be interpreted as the square of a correlation length, since the classical flat-space arc-length, $\xi \propto \int\left(g_{i j} \dot{x}^{i} \dot{x}^{j}\right)^{1 / 2} d t$, must be corrected by $\sim R / \Omega, \Omega$ the mesoscopic volume of the discretized path integral, when the volume element becomes sufficiently small [26]. This interpretation is intimately connected to the generalization of the Onsager-Machlup result [13], by observing that the factor $g^{1 / 2}$ is related to the inverse Jacobian of the transformation, to the mesoscopic-macroscopic variables from the phase space of fluctuations of endpoints of the folded short-time propagators. Note that $L_{\Psi}$ is equivalent to the Feynman Lagrangian with its associated variational principle. This is not equivalent to specifying the Lagrangian of the WKB propagator of the most-probable path [5,7], which differs essentially in having the van Vleck determinant in the prefactor of the propagator, and in having a Lagrangian containing $R /\left(12 \mu_{m}\right)$ instead of $R /\left(6 \mu_{m}\right)$.

\section{NUCLEAR FORCES CURVATURE SCALAR}

To proceed with the calculation of the curvature scalar, the arc length in isotropic form is defined by

$$
d s^{2}=(1+W)^{-1}\left(d r^{2}+r^{2} d \theta^{2}+r^{2} \sin ^{2} \theta d \phi^{2}\right) .
$$

This can be put into "standard form," [27] which is more convenient for further calculation.

$$
\begin{aligned}
d s^{2} & =g_{i j} d x^{i} d x^{j}, \\
& =A\left(r^{\prime}\right) d r^{\prime 2}+r^{\prime 2}\left(d \theta^{2}+\sin ^{2} \theta d \phi^{2}\right), \\
r^{\prime 2} & =[1+W(r)]^{-1} r^{2}, \\
A\left(r^{\prime}\right) & =\left\{1-\frac{r}{2} W(r)_{, r}[1+W(r)]^{-1}\right\}^{-2}, \\
{[\cdots]_{, r} } & =\partial[\cdots] / \partial r .
\end{aligned}
$$

Using

$$
\begin{aligned}
& R_{r^{\prime}}^{r^{\prime}}=\left(r^{\prime} A^{2}\right)^{-1} A_{, r^{\prime}}, \\
& R_{\theta}^{\theta}=R_{\phi}^{\phi}=r^{\prime-2}+\left(2 r^{\prime} A^{2}\right)^{-1} A_{, r^{\prime}}-\left(r^{\prime 2} A\right)^{-1}, \\
& A_{, r^{\prime}}=A_{, r} r_{, r^{\prime}}
\end{aligned}
$$

yields

$$
R(r)=\frac{2}{r^{\prime 2}}\left[1-\frac{1}{A\left(r^{\prime}\right)}+\frac{r^{\prime} A\left(r^{\prime}\right)_{, r^{\prime}}}{A\left(r^{\prime}\right)^{2}}\right] .
$$

The potential used to determine the size of $R$ is taken from the earliest paper that developed a momentum-dependent potential based on one-meson-exchange Born amplitudes, the set of $\{\pi, \eta, \sigma, \rho, \omega, \phi\}[19]$. Although this potential had moderate success in fitting the nonrelativistic scattering data, the deuteron, and nuclear matter, as well as being useful for neutron stars [28], with much fewer parameters than its static predecessors, certainly a more updated potential, e.g., including meson inelasticities and off-shell effects, should eventually be used to more precisely calculate $V$ and $R$. However, this potential is much easier to handle, algebraically and numerically, and the resulting calculations are 
certainly within the correct order of magnitude.

The potential taken here is the sum of ${ }^{1} S_{0}$ and ${ }^{3} S_{1}$ states, the latter without including coupling to the ${ }^{3} D_{1}$ state. This is not a serious approximation for this momentum-dependent potential, since it was shown $[19,20]$ that the fitted coupling is much less than in previous static potentials. This permits the isotropic assumption made above. $r \times p \cdot S$ (Spin-Orbit) and $\left(3 \sigma_{1} \cdot \hat{r} \sigma_{2} \cdot \hat{r}-\sigma_{1} \cdot \sigma_{2}\right)$ (Tensor) amplitudes are zero in these states, leaving 1 (Central), $\sigma_{1} \cdot \sigma_{2}$ (Spin-Spin), and $\frac{1}{2 m^{2}}\left(\sigma_{1} \cdot p \sigma_{2} \cdot p+\sigma_{2} \cdot p \sigma_{1} \cdot p\right)$ to be considered; in $S$-states the latter reduces to $\frac{1}{3 m^{2}} \sigma_{1} \cdot \sigma_{2} p^{2}$ [19].

The parameters used to fit the potential [19] are: $g_{\pi}^{2}=14.0, \mu_{\pi}=0.135$ (all masses given in $\left.\mathrm{Gev}^{-1}\right), g_{\eta}=14.0, \mu_{\eta}=0.548, g_{\sigma}^{2}=3.036, \mu_{\sigma}=0.461, g_{\rho 1}^{2}=1.1$ (vector coupling), $g_{\rho 2}^{2}=21.9$ (tensor coupling), $g_{\rho 12}^{2}=g_{\rho 1} g_{\rho 2}$ (mixed vector-tensor coupling), $\mu_{\rho}=0.531, g_{\omega}^{2}=g_{\phi}^{2}=3.03, \mu_{\omega}=0.78$, and $\mu_{\phi}=1.02$. The artificial necessity of including the $\sigma$ and the low mass of the $\rho$ were important factors in determining that much work remained to be done on the $\pi-\pi$ and the $\pi-\pi-\pi$ regions [20]. To cut off the artificial $r^{-3}$ divergence of the Spin-Orbit and Tensor forces, all these amplitudes were multiplied by $[1-\exp (-m r)]^{3}$. Not included here, because of computer restrictions, was the additional regularization of powers of $\left(q-q^{\prime}\right)^{n}$ in the $t$-channel $(n=0,2,4,6)$, before Fourier transforming to $r$-space, by $\prod_{i=1}^{3}\left(\Lambda_{i}^{2}-\mu^{2}\right) /\left[\Lambda_{i}^{2}+\left(q-q^{\prime}\right)^{2}\right]$, where all $\Lambda_{i}>m$. As with the former, the latter cutoffs also affect the potential near the core region, but they die off soon afterwards, e.g., after 3 to $4 \mathrm{Gev}^{-1}$, relative to the contributions being made by the exchanged mesons. Instead, here $\left(q-q^{\prime}\right)^{n} \rightarrow\left(-\mu^{2}\right)^{n / 2}$.

\section{-- Fig. 1 --}

Figure 1 presents the results. Note that the ratio, $R /\left(6 m v_{s}\right)$ in (d) is substantial throughout the main potential region, to eventually approach its asymptote at $-\frac{1}{6}\left(\mu_{\pi} / m\right)^{2}=-0.00345$.

\section{CONCLUSION}

It now is clear that these Riemannian corrections are small, but not negligible. For example, if a velocity-dependent potential were fit to data using the Schrodinger equation, this potential would have to include the curvature term. The parameters of this fit most likely would absorb this affect so that the net functional form would be only slightly affected. However, in calculating the nuclear matter $K$ matrix, which is derived from a Lagrangian or Hamiltonian path-integral representation, this curvature term would not appear, thereby directly affecting the binding energy and saturation properties. A correction on the order of $1 \%$ in the potential could mean on the order of $1 \mathrm{Mev}$ correction to the binding energy ( a cancellation of the kinetic energy and the self-consistent nuclear matter potential), especially since this correction is influential in the steep part of the potential presented in Fig. 1 [19,20].

It would be interesting to see what $R$-effects exist in hadrons, e.g., as modeled by velocity-dependent quark-quark potentials.

\section{ACKNOWLEDGMENTS}

I thank Dirk Roekaerts for clarifying several issues in the definitions of the various short-time propagators. Calculations and plots were facilitated with the PDP-10 MACSYMA Consortium algebraic manipulator at the Massachusetts Institute of Technology, supported in part by USERDA E(11-1)-3070 and NASA NSG 1323. This project has been supported entirely by personal contributions to Physical Studies Institute and to the University of California at San Diego Physical Studies Institute agency account through the Institute for Pure and Applied Physical Sciences.

\section{REFERENCES}

1. B.S. DeWitt, Dynamical theory in curved spaces. I. A review of the classical and quantum action principles, Rev. Mod. Phys. 29, 377-397 (1957).

2. K.S. Cheng, Quantization of a general dynamical system by Feynman's path integration formulation, J. Math. Phys. 13, 1723-1726 (1972). 
3. L.S. Schulman, Techniques and Applications of Path Integration, J. Wiley \& Sons, New York, (1981).

4. J.D. Bekenstein and L. Parker, Path integrals for a particle in curved space, Phys. Rev. D 23, 2850-2869 (1981).

5. R. Graham, Lagrangian for diffusion in curved phase space, Phys. Rev. Lett. 38, 51-53 (1977).

6. U. Weiss, Operator ordering schemes and covariant path integrals of quantum and stochastic processes in curved space, Z. Phys. B 30, 429-436 (1978).

7. F. Langouche, D. Roekaerts, and E. Tirapegui, Functional Integration and Semiclassical Expansions, Reidel, Dordrecht, The Netherlands, (1982).

8. F. Langouche, D. Roekaerts, and E. Tirapegui, Discretization problems of functional integrals in phase space, Phys. Rev. D 20, 419-432 (1979).

9. H. Dekker, Quantization in curved spaces, in Functional Integration: Theory and Applications, (Edited by J.P. Antoine and E. Tirapegui), pp. 207-224, Plenum, New York, (1980).

10. T. Kawai, Quantum action principle in curved space, Found. Phys. 5, 143-158 (1975).

11. R. Graham, Covariant formulation of non-equilibrium statistical thermodynamics, Z. Physik B26, 397-405 (1977).

12. H. Grabert and M.S. Green, Fluctuations and nonlinear irreversible processes, Phys. Rev. A 19, 1747-1756 (1979).

13. H. Grabert, R. Graham, and M.S. Green, Fluctuations and nonlinear irreversible processes. II., Phys. Rev. A 21, 2136-2146 (1980).

14. H. Dekker, Functional integration and the Onsager-Machlup Lagrangian for continuous Markov processes in Riemannian geometries, Phys. Rev. A 19, 2102-2111 (1979).

15. L. Ingber, Statistical mechanics of neocortical interactions. I. Basic formulation, Physica $D$ 5, 83-107 (1982).

16. L. Ingber, Statistical mechanics of neocortical interactions. Dynamics of synaptic modification, Phys. Rev. A 28, 395-416 (1983).

17. L. Ingber, Statistical mechanics of nonlinear nonequilibrium financial markets, Math. Modelling 5 (6), 343-361 (1984).

18. L. Ingber, Riemannian corrections to velocity-dependent nuclear forces, Phys. Rev. C 28, 2536-2539 (1983).

19. L. Ingber, Nuclear forces, Phys. Rev. 174, 1250-1263 (1968).

20. L. Ingber and R. Potenza, Nuclear forces and nuclear energetics, Phys. Rev. C 1, 112-122 (1970).

21. M. Lacombe, B. Loiseau, J.M. Richard, R. Vinh Mau, J. Cote, P. Pires, and R. de Tourreil, Parameterization of the Paris N-N potential, Phys. Rev. C 21, 861-873 (1980).

22. W.R. Frazer, L. Ingber, C.H. Mehta, C.H. Poon, D. Silverman, K. Stowe, and H. Yessian, High energy multiparticle reactions, Rev. Mod. Phys. 44, 284-319 (1972).

23. M.M. Nagels, T.A. Rijken, and J.J. de Swart, Low-energy nucleon-nucleon potential from Reggepole theory, Phys. Rev. D 17, 768-776 (1978).

24. H. Toki, Short range behaviour of the N-N potential within the quark model, Z. Phys. A 294, 173-181 (1980).

25. F. Langouche, D. Roekaerts, and E. Tirapegui, Short derivation of Feynman Lagrangian for general diffusion process, J. Phys. A 113, 449-452 (1980).

26. G. Ruppeiner, New thermodynamic fluctuation theory using path integrals, Phys. Rev. A 27, 1116-1133 (1983).

27. S. Weinberg, Gravitational Cosmology, Wiley, New York, (1972).

28. J.R. Buchler and L. Ingber, Properties of the neutron gas and applications to neutron stars, Nucl. Phys. A 170, 1-11 (1971). 


\section{FIGURE CAPTION}

FIG. 1. The abscissa in all Figures is the $r$-axis, from $2 \mathrm{Gev}^{-1}$ to $6 \mathrm{Gev}^{-1}$. (a) The curvature contribution to the Schrodinger equation, $R /\left(12 \mu_{m}\right)$, versus $r$. (b) The static part of the potential (without terms from the symmetrized $\mathrm{W}$ contribution), $v_{s}$, versus $r$. (c) The momentum-dependent part of the potential, $W$, which contributes to the metric $g_{i j}$, versus $r$. (d) The ratio of (a):(b), $R /\left(6 m v_{s}\right)$ versus $\mathrm{r}$. 